\title{
Intelligent Analysis of Computer-Aided Garment Design CAD Based on Virtual Reality Model for Surplus Fabric Removal and Reuse without Segmentation of Cutting Pieces
}

\author{
Tanqiu Wang (iD \\ School of Humanities and Arts, Zhengzhou Technology and Business University, Zhengzhou 451400, China \\ Correspondence should be addressed to Tanqiu Wang; 2009030010@students.stamford.edu
}

Received 17 November 2021; Revised 18 December 2021; Accepted 27 December 2021; Published 13 January 2022

Academic Editor: Qiangyi Li

Copyright (c) 2022 Tanqiu Wang. This is an open access article distributed under the Creative Commons Attribution License, which permits unrestricted use, distribution, and reproduction in any medium, provided the original work is properly cited.

\begin{abstract}
For the purpose of improving the efficiency of garment design, the computer-aided garment design virtual reality (VR) model for surplus fabric removal and reuse without segmentation of cutting pieces is analyzed in this paper to provide the architecture of the computer-aided garment design CAD system. The form of dividing the garment into multiple types of nonsegmented pieces is adopted so that each nonsegmented piece stands for a complete design element unit. Based on this structure, the computer analysis of garment design based on CAD can be connected at a deeper level, which will not only improve the design efficiency of new garments but also reduce the design time at the client terminal and enhance the quality of the design. Through the experimental operation of prototypes, it is verified that the intelligent system proposed in this paper can implement the design of prototypes quickly and effectively.
\end{abstract}

\section{Introduction}

Currently, the garment industry has been through major reforms, and the garment is produced in small and largescale customized form with a number of varieties. The socalled mass production refers to a model where the garment is produced on a large scale based on the special requirements of customers to supply customized garments and services at a lower cost with high quality and high efficiency $[1,2]$. This model ensures that rapid garment design can maintain a good standard. Computer-aided CAD intelligence complies with the development demand of garments and can provide enterprises with a favorable competitive edge and respond to market changes quickly. Hence, it has been extensively applied in the garment industry at present. CAD garment intelligent technology provides enterprises with advanced product design. Certainly, the rapid increase of design resources in an explosive form has also brought about new problems. For example, there are a large number of data formats, poor performance in data sharing, redundant data, and waste of resources; it is difficult to browse, consult, and revise the information; there are a lack of reliability and poor security performance.

Computer CAD controls all the information associated with the product, including product specifications, electronic files, CAD files, product standards, and storage time. It also covers the processes corresponding to the product, such as the drawing review, issuance, alterations, and other technical issues [3]. With regard to the virtual reality model for surplus fabric removal and reuse without segmentation of cutting pieces, it is a prerequisite for garment design. Compared with the previous design data, the technique without segmentation of cutting pieces can offer greater advantages to garment design and development. The data stored in this model can demonstrate all the knowledge in the whole process to implement acquisition, sharing, and repeated application. In this paper, the intelligent combination of computer CAD and garment design is implemented based on the background of the development of the virtual reality model for surplus fabric removal and reuse without segmentation of cutting pieces, which can improve the design level and knowledge management capacity of the garment industry in all aspects. 


\section{Garment Design Virtual Reality Model for Surplus Fabric Removal and Reuse without Segmentation of Cutting Pieces}

In the garment design industry at present, for the purpose of meeting the different requirements of customers, it is necessary to respond to their individual demands in a timely manner, which is mainly divided into two links, that is, the development and the design of garment. On the basis of a full analysis of the changes in market needs, personalized services can be provided to customers based on the emerging garment development processes and design cycles; at the same time, the personalized garment can be designed in accordance with the actual requirements of customers. The goals of these two links are not the same as those of the primary tasks. However, there is an inherent connection between them. Modularization and standardization in the process of garment design are taken as the foundation of the garment design process. With regard to the transformation of the nonsegmented pieces of garment in the garment design process into a virtual reality model for surplus fabric removal and reuse, the knowledge to support the development of new garments and the knowledge of customer design based on customer requirements should be included at the same time [4]. Figure 1shows the garment development and design process from the nonsegmented pieces to the virtual reality model for surplus fabric removal and reuse.

The virtual reality model of no segmentation of cutting pieces to surplus fabric removal and reuse (VRMNSEP) is designed with the development of virtual reality to "enhance" the real world by superimposing computer-generated virtual objects, scenes, or system prompt information on the actual scenes. The virtual reality model for surplus fabric removal and reuse without segmentation of cutting pieces can not only introduce the virtual object into the real environment but also provide the position and posture of the virtual object dynamically to maintain the consistency of the virtual object with the actual scene and help form the habit of roaming in the virtual reality model environment based on observation in the real world. This way of interaction seems more natural. On the other hand, as the real scene can be maintained in the virtual reality model for surplus fabric removal and reuse without segmentation of cutting pieces, the output result will be more realistic.

As shown in Figure 2, the video stream can be obtained by using the camera to shoot the real situation based on the virtual reality model for surplus fabric removal and reuse without segmentation of cutting pieces. Each frame in the video stream is processed by using the tracking method, and the actual coordinates and state of the camera are calculated based on the geometric calculation methods. The coordinates and states (also known as 3D registration) of the virtual objects registered in the actual background are used to establish a virtual scene. The virtual scene and the real background video stream are combined by using the technology of video combination, and the combined result (video stream) is transmitted to the display in a timely manner $[5,6]$.
In the case of a virtual reality model system for surplus fabric removal and reuse without segmentation of cutting pieces of garment, the most important part is to add virtual objects to the actual scene in real time and align them with real world objects correctly [7].

After the direction of each axis is identified, the origin is set on the axis. The overall structure of the garment should be symmetrical (Figure 3). This system requires the application of CAD technology for the rendering of the garment. In the envisaged scene, the various lines that consist of the garment should meet a certain ratio so that the garment on the screen can become more realistic. Hence, it is necessary to zoom in and out on the garment in a certain proportion.

In reference to the design drawing of the garment, it is zoomed in and out at a certain proportion in the system. When the outer wall of the garment is implemented, the solution is to attach lines with consistent specifications. The department based on the length and width of a line can be taken to address the problem of scale based on the overall lines and blocks of the garment. When the construction of the coordinate system and the scale is completed, the geometric entity or geometric entity of the virtual garment can stand for each line that constitutes the garment established by a series of calculations. Each line has two most basic attributes, that is, position and parameter. As shown in equation (1), the mathematical modeling of lines is carried out:

$$
\text { construction (pos, VRMNSEPgs). }
$$

The construction in equation (1) stands for components. pos stands for the position of the part, and VRMNSEPx stands for the parameter of the component.

The position of the component is the coordinate, and the coordinate is the space rectangular coordinate system. The point with the smallest coordinate is selected based on the priority of the $z$-axis, the axis, and the $y$-axis. In other words, the point with the smallest $z$-axis value is selected from the points of all parts [8]. If there is no more than one point, the point with the smallest $x$-axis value is selected. If there are repeated points, the point with the smallest $y$-axis value is selected, and this point is the location of this component. The parameters of a part are a series of values, which stand for the shape information of the part. When a part is a cuboid, the parameters of the part include the length, width, and height of the part. After completion of the modeling, the overall garment can be the result of a series of requirements and operations on the parts. The mathematical modeling of the garment is being demonstrated at present.

$$
\text { library }=\sum \text { construction (pos, args). }
$$

In the simulated costume scene described in this paper, the costume is from a certain school. The garment style is symmetric and standardized. Hence, that material is basically various combinations of rectangles. In this paper, the garment in front is taken as an example to introduce the modeling process of the materials. The $3 \mathrm{D}$ visualization modeling of garment heritage is shown as follows: 


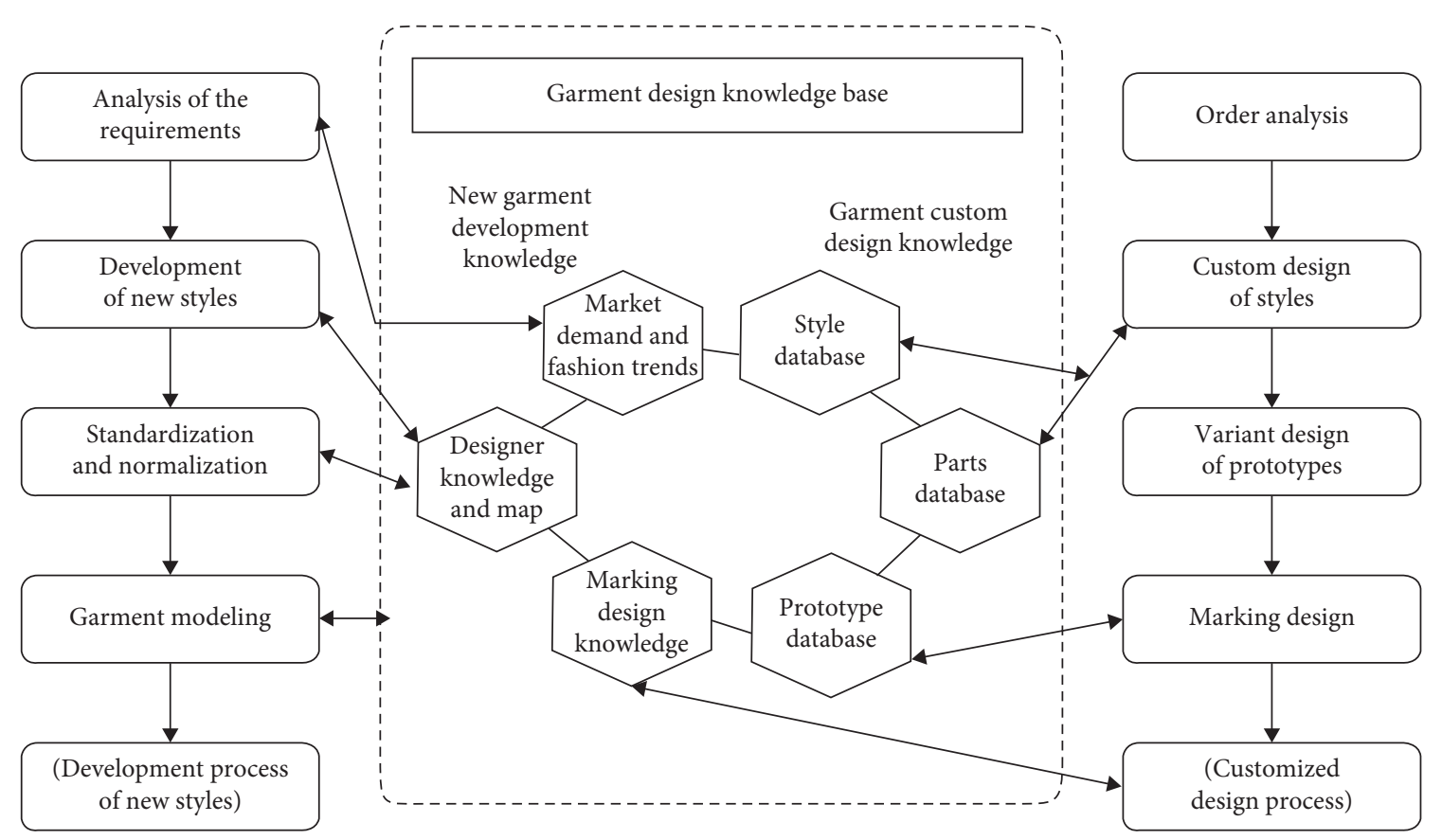

FIGURE 1: Garment design process.

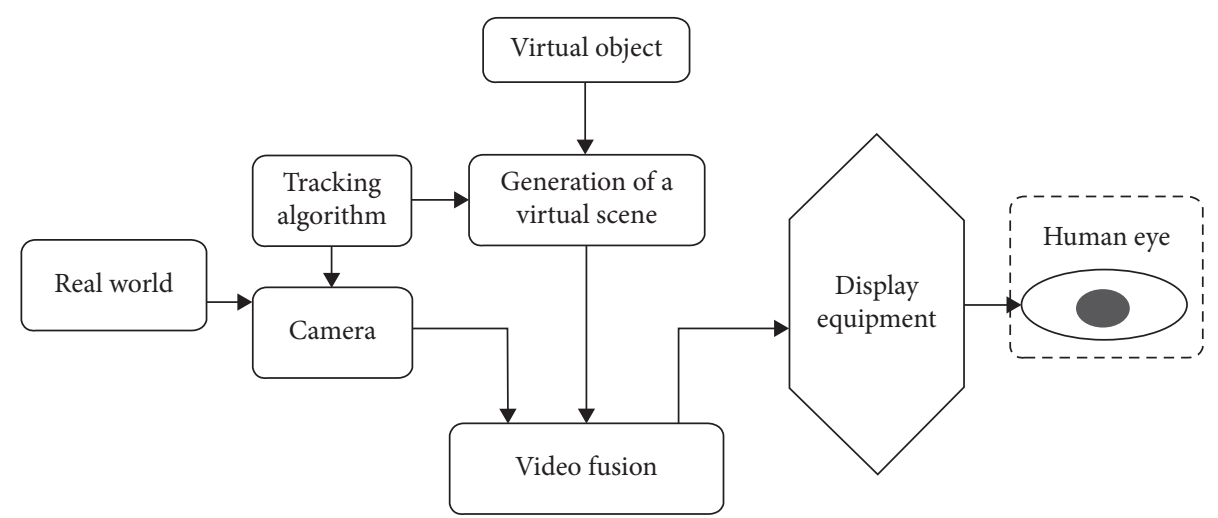

FIGURE 2: Diagram of the virtual reality model for surplus fabric removal and reuse without segmentation of cutting pieces.

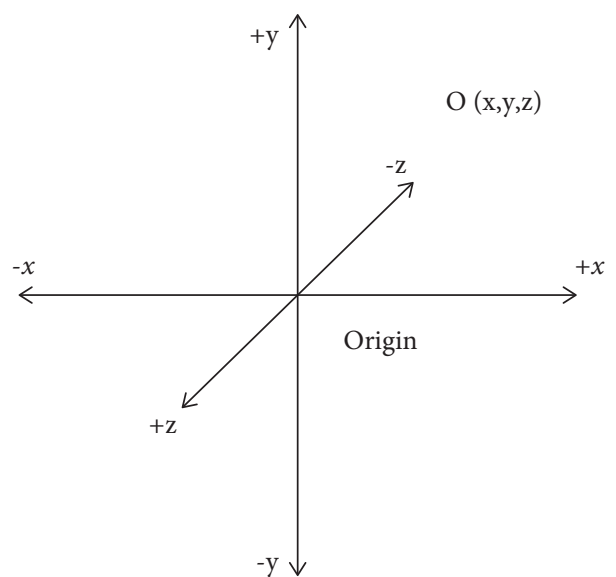

Figure 3: Cartesian space coordinate system diagram. 
stairs (pos, len, wid, hei, num).

In the above formula, pos stands for the position coordinate of the garment part. nstands for the length of the garment, wid stands for the width of the garment, hei stands for the height of the garment, and num stands for the total number of garment grades.

In this section, the various stages of the garment are deemed as a whole rectangular body, while the overall garment is stacked with rectangles with various heights. Based on the above expression, pos stands for the coordinate of the starting rectangle, and the height of the rectangle width is also the value for the height of the input width. The second rectangle is the second rectangle of num. Adding the width to the $z$ value of the starting coordinate is the position coordinate. With regard to this parameter information, the length and width of the cuboid are constant, and the height is accumulated [9]. It can also be understood that the $z$ value for the coordinate of each rectangular parallelepiped member and the height value of the parameter in the step are continuously changed as an arithmetic sequence, with the equations being shown as follows:

$$
\begin{aligned}
& \text { stairs }=\sum_{i}^{\text {num }} \operatorname{Cubei}\left(\operatorname{pos}_{i}, \text { len, wid, hei } i_{i}\right. \text {, } \\
& \operatorname{pos}_{i} \cdot z=\operatorname{pos}_{1} \cdot z+(i-1) \cdot \text { wid, } \\
& \text { hei }_{i}=\text { hei }+(i-1) \cdot \text { hei. }
\end{aligned}
$$

The analysis of market demand is the first step in the development of new garment products. Through the analysis, enterprises can have a comprehensive understanding of the market demand and win more customers. Subsequently, it is necessary to forecast the fashion trend, correctly grasp the fashion trend, and lead the fashion trend. Popular trends include popular designs, fashionable colors, fabrics with good quality, and popular patterns. In addition, both inspiration and creativity are essential for designers to design new garments. The design of the new garment and the transformation of the corresponding nonsegmented pieces of garment to the virtual reality mode have an interactive relationship. In the process of garment design, it is necessary to combine the knowledge base according to the market demand and fashion status; the garment designers also need to adhere to the market development trend and fashion trend that they have understood as a database for design inspiration. The corresponding design styles are identified through the design pattern, and the design inspiration is integrated into the knowledge base [10]. After the design is completed, the garment design for the new product is transformed into design knowledge to meet the individualized requirements of garment customers. In the whole process of garment design, the requirements of customers on the garment design can mainly be divided into the customization of the garment, the variant design of the prototype, and the selection of garment fabric. After the customer places an order, the designer identifies the appropriate design style from the knowledge base and carries out the design and customization based on the requirements of the customer. After the design order is completed, the variant design of the prototype is carried out according to the body figure of the customer. Finally, the marker layout design is completed. The result of the design order, the variant design result of the prototype, and the design of the marking layout can be saved from the nonsegmented pieces to the virtual reality model of surplus fabric removal and reuse for the subsequent application.

\section{Overall System Design and Function}

For the purpose of completing the design of a piece of garment, this system consists of two parts, that is, system module and user module. In the system module, the nonsegmented piece database of the garment is generally added and revised. The user module is used to design the garment and consists of the following parts: the design of the intelligent splines, the adjustment of detailed styles, the adjustment of the overall style, the filling of fabric, and the covering of the dress form, and so on. The specific process for the design of a piece of garment is shown in Figure 4.

The main functions of the user module are as follows.

SMAPTING of styles: each piece of garment is connected to a complete garment without segmentation. There are two ways of connection, that is, automatic and manual connection. Automatic splicing is that the user selects the preferred nonsegmented garment from various types of garment series that have already been completed in the system to create a new style. The manual spline is that the user directly selects the appropriate nonsegmented pieces table from the nonsegmented piece database to perform an exchange.

Adjustment of design details: after users complete the rough design of the garment, if they are still not satisfied with the details of the cutting pieces in the garment, they can adjust the details of each piece as needed. At this stage, the system also offers two methods. The user can directly edit the key points or select the trend of the piece style to shift toward the key points by the system automatically. Adjustment of overall style: make the adjustment to the top and bottom. The top and bottom can be designed by the user or the design stored in the system. In either case, it should be a reasonable stacking of the top and bottom [11].

Filling of the fabric and covering of the dress form: in the previous stages, the design line is designed. In the fabric filling stage, users can fill in different fabrics to cover the dress form, which can help users gain a more perceptual understanding of the garment design so that they can evaluate its advantages and disadvantages more directly. At the same time, the user offers a system database or a user database. The system database is provided to the system. The garment made by professional costume designers in advance has no segmented pieces, including the garment and the stacking of the top and the bottom.

In the garment design based on the virtual reality model for surplus fabric removal and reuse without segmentation of cutting pieces, the primary problem is to acquire the location state of the practical object. This is the prerequisite for implementing the location and registration of the virtual 


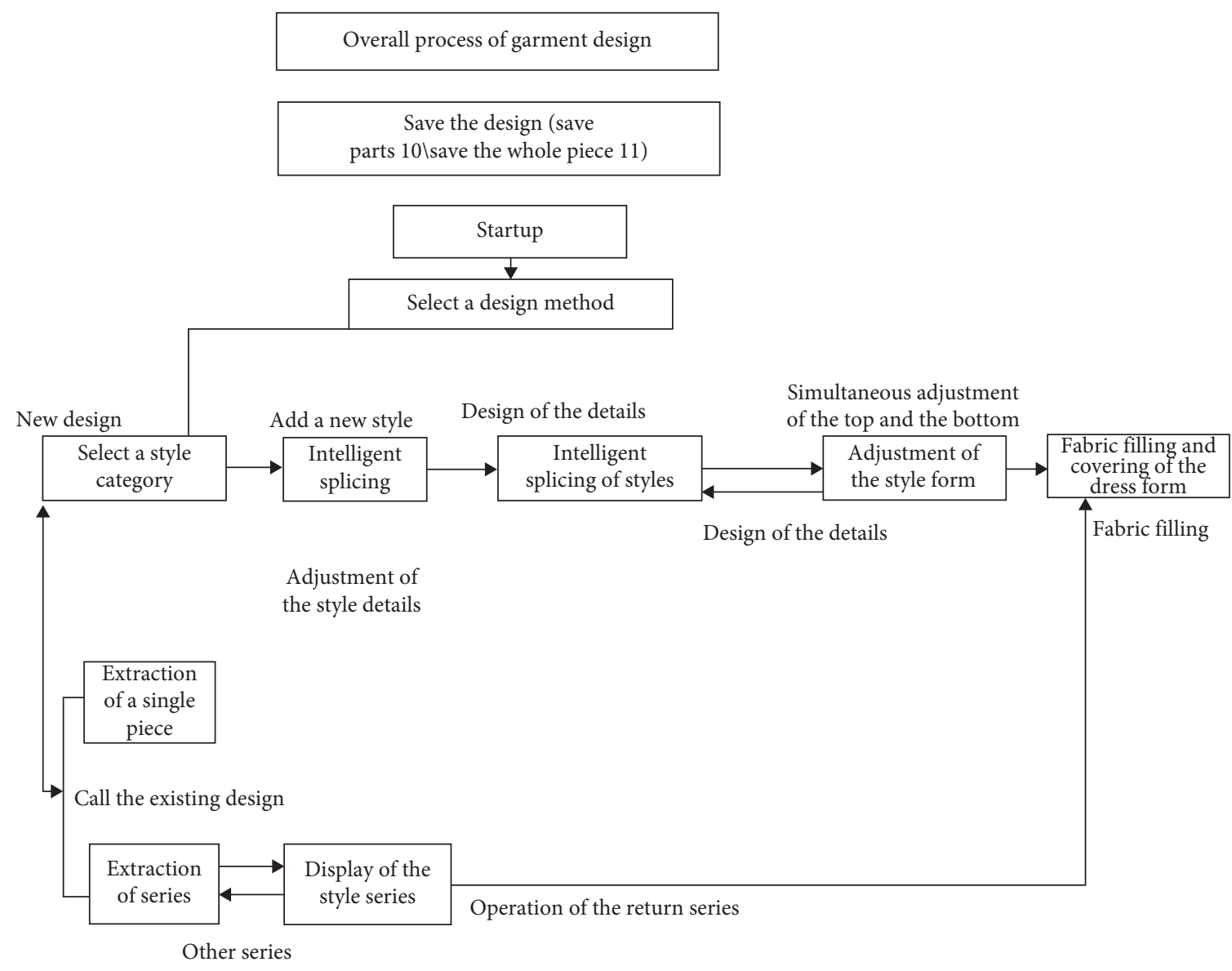

FIgURE 4: User module design process.

object and the key to realizing the satisfactory effect of the real environment. In the system, the application of markers to locate practical targets is not irregular positioning. The advantages of the former include simple tracking methods, high efficiency, and excellent results in practice.

The purpose of tracking the position of the practical object based on the mark card is to determine the position and posture of the mark card for the camera according to any one of the image sequences taken by the camera. Since the mark card is fixed to the tracking object, the position and posture of the mark card stand for the position and posture of the real object. In the real object position tracking algorithm of the marker card, the camera coordinate system, the $2 \mathrm{D}$ coordinate system of the photographing plane, and the marker card coordinate system are correlated with three coordinate systems (in reference to Figure 5).

Based on the principle of pinhole imaging, there is the following transformation relationship between the mark card coordinate system and the camera coordinate system.

$$
\left[\begin{array}{c}
X_{c} \\
Y_{c} \\
Z_{c} \\
1
\end{array}\right]=\left[\begin{array}{cccc}
U_{11} & U_{12} & U_{13} & V_{x} \\
U_{21} & U_{22} & U_{23} & V_{y} \\
U_{31} & U_{32} & U_{33} & V_{z} \\
0 & 0 & 0 & 1
\end{array}\right]\left[\begin{array}{c}
X_{m} \\
Y_{m} \\
Z_{m} \\
1
\end{array}\right]=\left[\begin{array}{cc}
U_{3 \times 3} & V_{3 \times 1} \\
0^{T} & 1
\end{array}\right]\left[\begin{array}{c}
X_{m} \\
Y_{m} \\
Z_{m} \\
1
\end{array}\right] .
$$

Equation (1) is a mark card camera transformation equation. In the equation, $\mathrm{U} 3 \times 3$ stands for the rotation matrix of the camera coordinate system for the mark card coordinate system; V $3 \times 1$ stands for the translation vector relative to the camera coordinate system of the mark card coordinate system. Hence, the problem is changed into the choice of the rotation matrix U3.

The coordinates below the camera coordinate system of a point above the mark card are set to $\left(X_{c}, Y_{c}, Z_{c}\right)$, and the coordinates below the $2 \mathrm{D}$ coordinate system on the camera surface of the point are set to $\left(x_{c}, y_{c}\right)$. Based on the imaging model of the camera, the following imaging equation for the camera should be met.

$$
\left[\begin{array}{c}
h x_{c} \\
h y_{c} \\
h \\
1
\end{array}\right]=\left[\begin{array}{cccc}
K_{11} & K_{12} & K_{13} & 0 \\
0 & K_{22} & K_{23} & 0 \\
0 & 0 & 1 & 0 \\
0 & 0 & 0 & 1
\end{array}\right]\left[\begin{array}{c}
X_{c} \\
Y_{c} \\
Z_{c} \\
1
\end{array}\right] .
$$

After shooting the two vertical edges A and B of the black rectangular frame in the mark card (as shown in Figure 6), the plane equation of the imaging plane coordinate system of the edge $A$ and edge $B$ can be the two vertical edges on the mark card, as shown in Figure 6. 


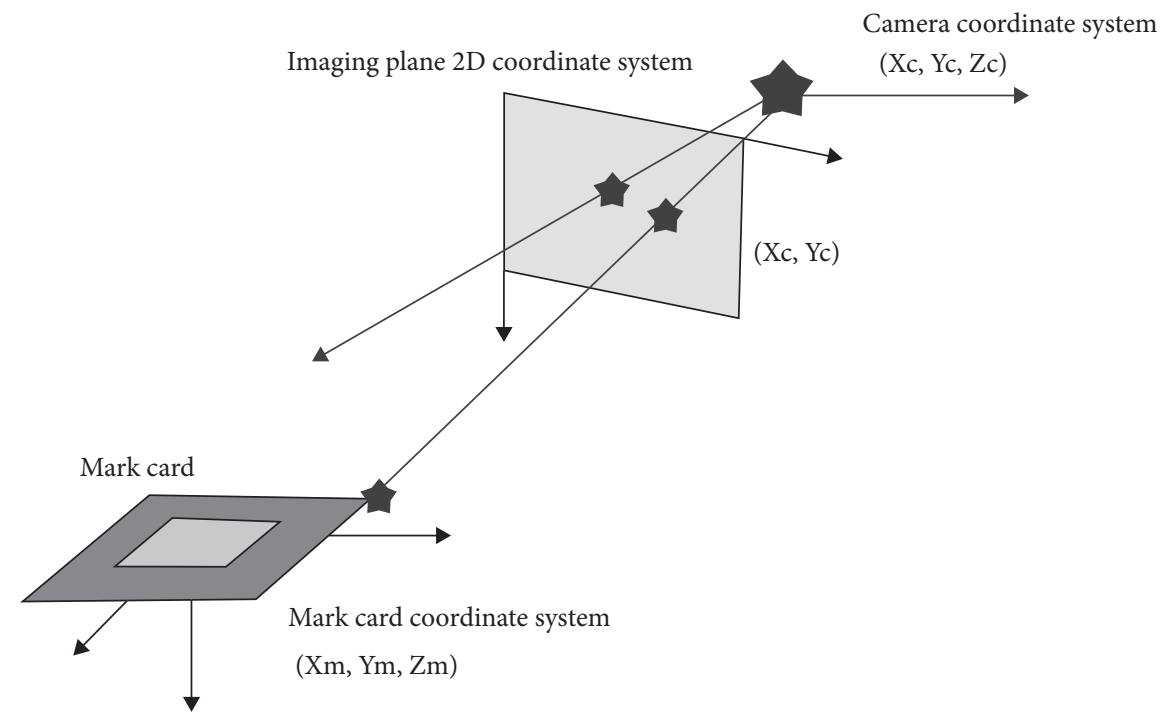

FIGURE 5: Relationship between the mark card coordinate system and the camera coordinate system.

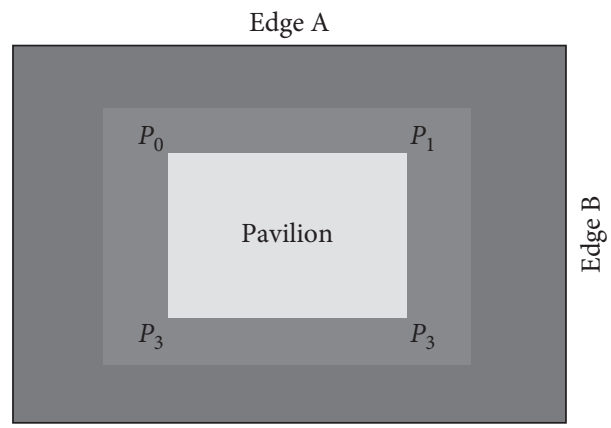

Figure 6: Black rectangular frame border of the photo mark card.

$$
\begin{aligned}
& a_{1} x+b_{1} y+c_{1}=0, \\
& a_{2} x+b_{2} y+c_{2}=0 .
\end{aligned}
$$

Equation (3) is introduced into equation (2) to obtain two vertical edge space equations for the rectangle as follows:

$a_{1} K_{11} X_{c}+\left(a_{1} K_{12}+b_{1} K_{22}\right) Y_{c}+\left(a_{1} K_{13}+b_{1} K_{23}+c_{1}\right) Z_{c}=0$,

$a_{2} K_{11} X_{c}+\left(a_{2} K_{12}+b_{2} K_{22}\right) Y_{c}+\left(a_{2} K_{13}+b_{2} K_{23}+c_{2}\right) Z_{c}=0$,

and the direction vectors of edge Aand edge Bare as follows:

$$
\begin{aligned}
& n_{1}=\left(a_{1} K_{11}, a_{1} K_{12}+b_{1} K_{22}, a_{1} K_{13}+b_{1} K_{23}+c_{1}\right), \\
& n_{2}=\left(a_{2} K_{11}, a_{2} K_{12}+b_{2} K_{22}, a_{2} K_{13}+b_{2} K_{23}+c_{2}\right) .
\end{aligned}
$$

As side Aand the side Bare also perpendicular to each other in space, the normal vector of the label card plane can be obtained as follows:

$$
n_{3}=n_{1} \times n_{2}
$$

$n 1, n 2$, and $n 3$ are unitized, respectively, to obtain 3 unit vectors that are perpendicular to each other, that is, $U 1, U 2$, and U3.

Thus, the rotation matrix can be obtained as follows:

$$
U_{3 \times 3}=\left[U_{1}^{t} U_{2}^{t} U_{3}^{t}\right]
$$

Equations (1), (2), and (5) are combined to obtain the system of equations as follows:

$$
\left[\begin{array}{l}
{\left[\begin{array}{c}
X_{c} \\
Y_{c} \\
Z_{c} \\
1
\end{array}\right]=\left[\begin{array}{cccc}
U_{11} & U_{12} & U_{13} & V_{x} \\
U_{21} & U_{22} & U_{23} & V_{y} \\
U_{31} & U_{32} & U_{33} & V_{z} \\
0 & 0 & 0 & 1
\end{array}\right]\left[\begin{array}{c}
X_{m} \\
Y_{m} \\
Z_{m} \\
1
\end{array}\right]=\left[\begin{array}{cc}
U_{3 \times 3} & V_{3 \times 1} \\
0^{T} & 1
\end{array}\right]\left[\begin{array}{c}
X_{m} \\
Y_{m} \\
Z_{m} \\
1
\end{array}\right],} \\
{\left[\begin{array}{c}
h x_{c} \\
h y_{c} \\
h \\
1
\end{array}\right]=\left[\begin{array}{cccc}
P_{11} & P_{12} & P_{13} & 0 \\
0 & P_{22} & P_{23} & 0 \\
0 & 0 & 1 & 0 \\
0 & 0 & 0 & 1
\end{array}\right]\left[\begin{array}{c}
X_{c} \\
Y_{c} \\
Z_{c} \\
1
\end{array}\right],} \\
U_{3 \times 3}=\left[\begin{array}{ll}
U_{1}^{t} U_{2}^{t} U_{3}^{t}
\end{array}\right] .
\end{array}\right.
$$

In equation (6), there are 8 unknown numbers, that is, $K 11, K 12, K 13, K 22, K 23, V x, V y$, and $V z$. Based on the imaging coordinates of the rectangular points P0P1, P2, and $\mathrm{P} 3$ of the rectangular frame and the coordinates of the mark card coordinate system for the cardinal points, the eight unknown points can be calculated. In this way, the translation vector of the camera coordinate system relative to the mark card coordinate system can be obtained. 


\section{Intelligent Analysis of Computer-Aided Garment Design CAD in Support of Rapid Design}

4.1. Support of CAD Intelligent Analysis of Garment Design for Custom Design of Styles. The goal of computer-aided garment design CAD intelligent analysis is to support the designers so that they can visualize the garment in their mind, develop a preliminary design prototype, and lay a solid foundation for the subsequent structural design of the garment, prototype production, and overall processing [12]. In the process of custom design of the garment, it is necessary to determine whether the design is consistent with the requirements of customers. If they are consistent, the design results can be output; otherwise, the relevant nonsegmented pieces will be selected from the nonsegmented piece database to form new garment styles. It allows revision based on the current model. Figure 7 shows the preparatory work for the satisfaction of the customers with the requirements of costume design.

In the process of conducting custom design, the intelligent analysis based on computer-aided garment design CAD in support of high-speed design should be able to facilitate the connection between different discontinuous seats. The design of modular garments can connect different combinations of various cutting pieces, such as collars, collars, and sleeves. The CAD intelligent analysis system should be able to extract the nonsegmented pieces from the nonsegmented piece warehouse easily to carry out connection. For example, there may be some special requirements for the correct docking of two corresponding parts. The intelligent analysis of computer-aided garment design CAD should reflect these special requirements. Nonsegmented piece database and custom design database in the virtual reality model for surplus fabric removal and reuse without segmentation of cutting pieces of garments are the basis of design custom design. The CAD intelligent analysis of garments is implemented through an interface for the nonsegmented pieces or design data, which need to be extracted quickly, accurately, and completely from the CAD intelligent analysis system.

4.2. Support of CAD Intelligent Analysis of Garment Design for Variant Design of Prototypes. For the purpose of implementing the variant design of prototypes, it is necessary to make the model for the basic prototype in the CAD intelligent analysis system, record the operation process of making the prototype model, and prepare the size specification table at the same time. The whole process of garment design can indicate the design process of the prototype, from which the total design process of the garment prototype can be observed. It is mainly used to modify the garment design operation and carry out the variant design of the garment prototype. The garment design size table can be used to record the different pattern layouts with different styles. In the garment customer design stage, the garment size can be modified based on the actual demand and the body figure of the customers [13]. At the same time, it can also be combined with the process records of garment prototype making. The
CAD intelligent model for the analysis of the prototype can be used to carry out the design of the prototype preferred by the customers.

4.3. Support of CAD Intelligent Analysis of Garment Design for Marker Layout Design. In the production of small batches of single SKU and large quantities of the garment, the types and batches ordered by customers can change frequently. As a result, the pattern layout diagram needs to be redesigned in the production process accordingly.

\section{Application of Virtual Reality Model for Surplus Fabric Removal and Reuse without Segmentation of Cutting Pieces in Garment Style Design}

The data management of garment products is implemented based on a certain mathematical model, which can effectively organize the relevant data of the product from garment design, engineering process design, manufacturing and processing, and sales maintenance to exit and improve product quality accordingly. In this way, the market launch of products can be accelerated, and the market competitiveness of garment products can be improved. For the purpose of achieving the above objectives, it is necessary to support high-speed garment design, improve garment design efficiency, and shorten the time needed so as to meet the demand of customers. The functions of the computer-aided garment design CAD intelligent analysis are shown in Figure 8 .

Garment structure design: it also referred to pattern making. In the pattern making of the traditional garment, the pattern maker directly makes the pattern on cowhide or fabric by using the pattern-making tools. In the 1990s, with the continuous development and advancement of garment CAD software functions, garment pattern makers have gradually become familiar with and accepted the garment structure design system. The CAD structure design system of garments offers a series of drawing tools. In recent years, due to the application of computer virtual reality models for nonsegmented piece transmission in this field, mutual transformation of $2 \mathrm{D}$ and $3 \mathrm{D}$ garment prototypes has been implemented.

5.1. Application in the Transformation of 2D Prototypes into $3 D$ Garment. Garment CAD can be used to transform a 2D garment prototype into a $3 \mathrm{D}$ prototype based on the $3 \mathrm{D}$ model and display technology from the nonsegmented pieces to the virtual reality model for surplus fabric removal and reuse. This technology is also referred to as $3 \mathrm{D}$ visual stitching technology [14]. For example, one of the functions of the AGMS software pattern-making system is to edit the plane prototypes in 3D points, select the people in demand, and finally stitch the prototypes in the virtual environment. After the virtual prototype is completed, it can be tried on accordingly. In the whole process, the pattern maker can have a dialogue with the product and revise the design in a 


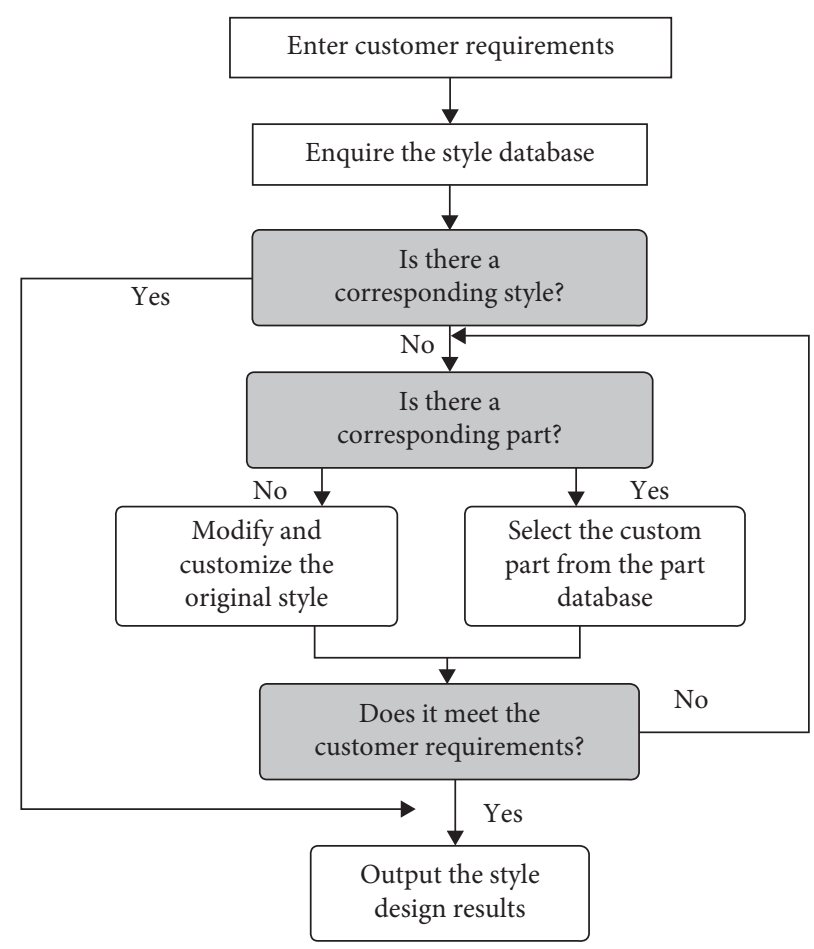

Figure 7: Custom design process of styles.

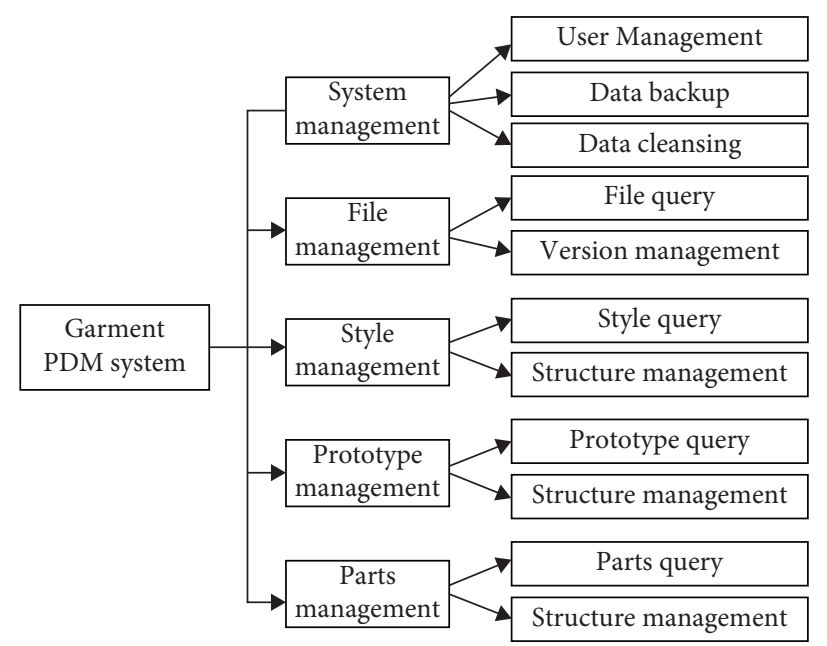

FIGURE 8: Function module of CAD intelligent analysis of garment design.

3D state until a completely satisfactory prototype of the garment is obtained, as shown in Figure 9.

5.2. Application in the Transformation of $3 D$ Garment into $2 D$ Prototypes. Different from the analysis above, CAD computer technology is used to transform the $3 \mathrm{D}$ garment into a $2 \mathrm{D}$ view of the same garment in the plane. Based on the prototype stripping function based on the intelligent CAD analysis of the garment, it is possible to make virtual production from the computer to the surface of the dress form, segment the garment prototypes on the surface of the dress form, and transform the highly fit garment into prototypes, which are then used as the basis to design garment prototypes, as shown in Figure 10.

5.3. CAD Integration Based on Knowledge Base. In order to design nonsegmented pieces, manage prototypes and operation process records, and implement the seamless system integration more effectively, the models, prototypes, nonsegmented pieces, and operation process records are taken as the objects [15]. The following master record objects are introduced into the computer-aided garment design CAD intelligent analysis. 


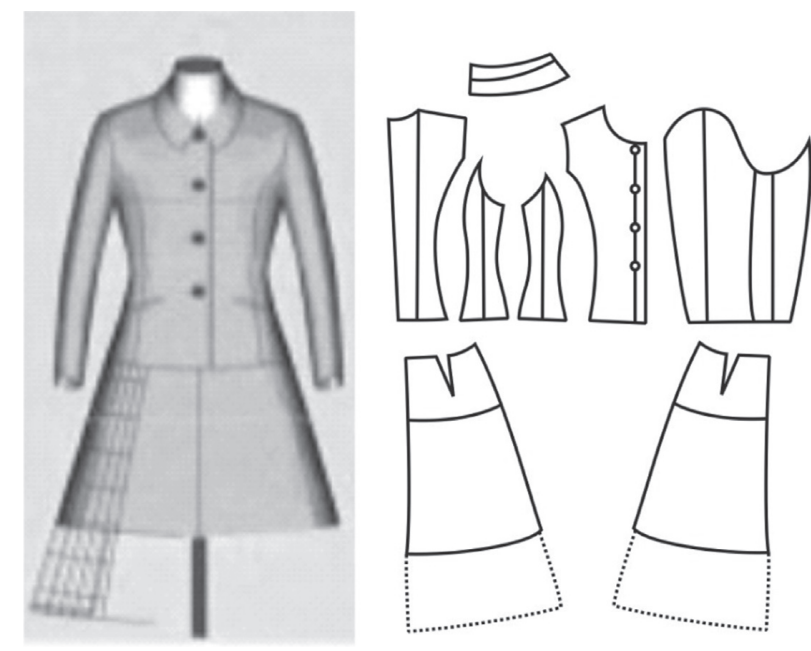

FIgURE 9: Transformation of 2D prototypes into a $3 \mathrm{D}$ garment in a virtual environment.
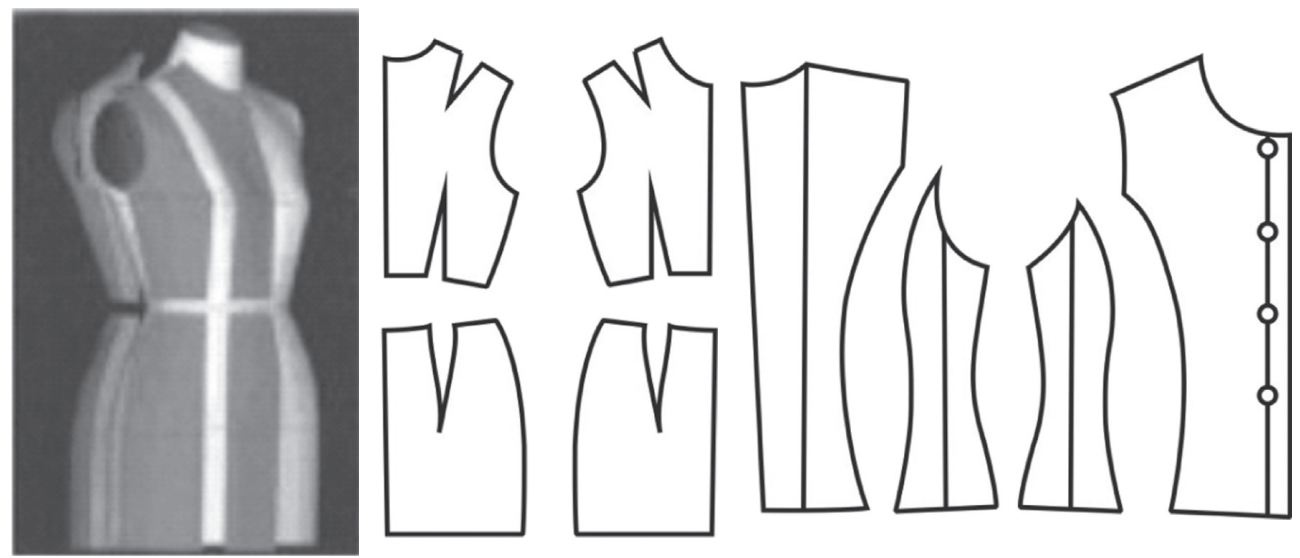

FIgURE 10: Transformation of a 3D garment into 2D prototypes in a virtual environment.

5.4. Master Record Object. Style master record (SMR): it refers to the data objects that describe the basic management features of styles in the process of garment design, including the style number, version number, processing status, creator, and other features.

Prototype master record (PMR): it refers to the data objects that describe the prototype management features in the prototype design, including the prototype number, version number, processing status, author, and other features.

Parts master record (PTMR): it refers to the data objects that describe the management features of the separator during the garment design process, including the nonsegmented piece number, version number, processing status, builder, and other features.

Process card master record (PCMR): it refers to the data objects that record the operating steps in the record files, which will be introduced in the following section, including the operation step record number, version number, processing status, author, and other features.

5.5. Logical Data Structure of System Integration. The integration of computer-aided garment design CAD intelligent analysis can be traced back to the overall design process, the management of the existing design, nonsegmented pieces, prototypes, and so on to ensure the integrity of data, improve the efficiency and quality of the total design process, and therefore enhance the overall interests of the enterprise. Figure 11 shows the logical data structure of the system integration based on the computer-aided garment design CAD intelligent analysis from the CAD intelligent analysis system and the nonsegmented pieces of garment to the virtual reality model for surplus fabric removal and reuse.

In the garment design process, the design knowledge of the designer and the results need to be saved from the nonsegmented pieces of garment to the virtual reality model for surplus fabric removal and reuse so that they can also be used by others, the relevant knowledge can be shared, and the personal knowledge can be transformed into corporate property. In addition, it is required that the design results should be related to CAD intelligent analysis. Hence, CAD intelligent analysis of garment design can be used to manage the overall design knowledge and process, improve the design efficiency and quality, reduce the design costs, and allow the designers to acquire the design knowledge needed more efficiently. 


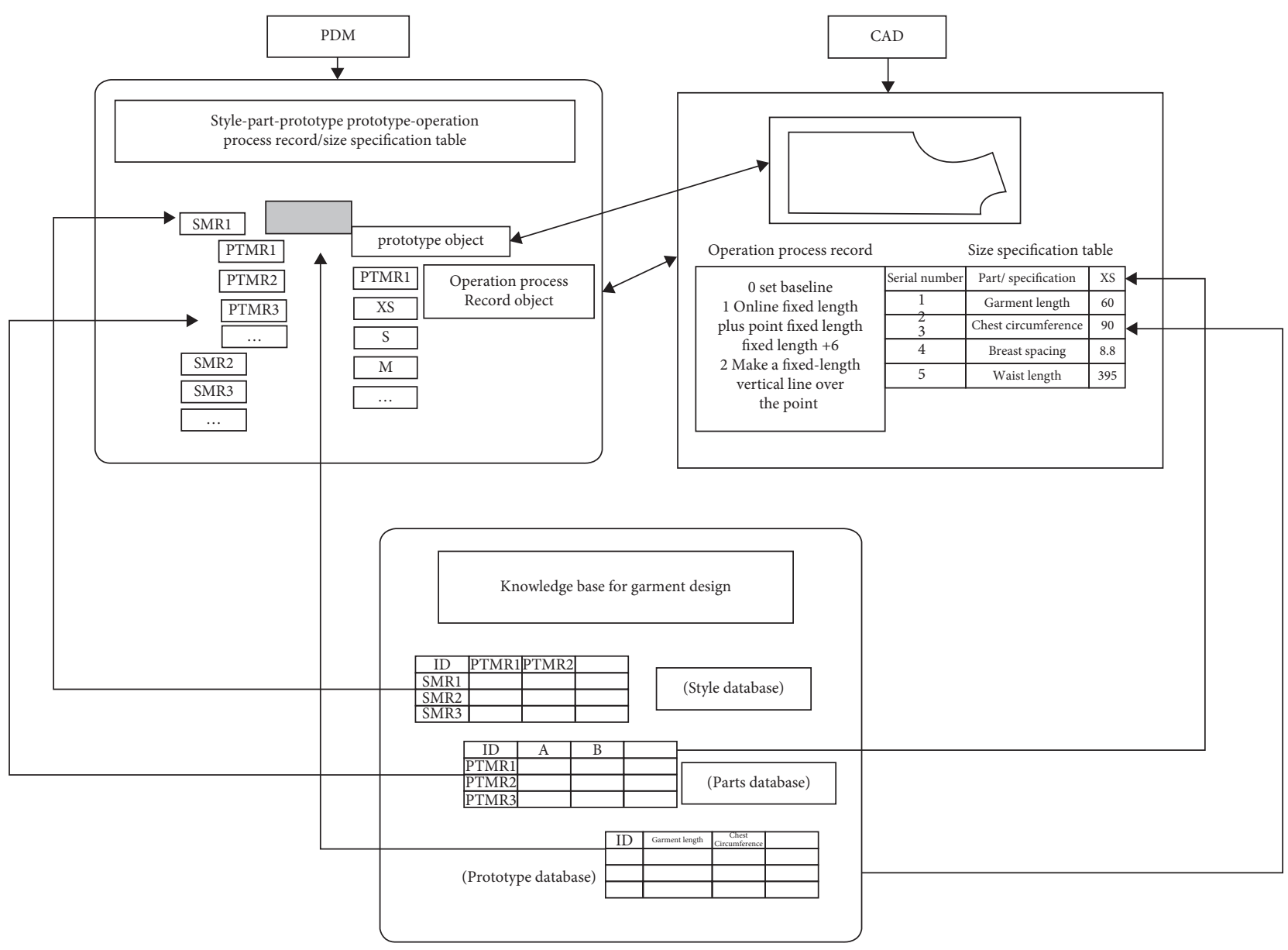

FIGURE 11: Logical data structure of system integration.

In the virtual reality model for surplus fabric removal and reuse without segmentation of cutting pieces of garment, the preauthorization, nonsegmented piece database, and the prototype database are managed in the form of an item characteristic table. Based on the object features table, the relevant models, nonsegmented pieces, and prototypes can be classified. In this way, the various features of models, nonsegmented pieces, and prototypes can be explained. In addition, it can also describe some types of structural relationships between various pieces. The newly developed knowledge mainly falls into the category of knowledge management, market demand status, trend forecast, and so on. Since the data exchange with computer-aided garment design CAD intelligent analysis is not huge, the knowledge base is not represented in the logical data structure of Figure 4 . The style object property table includes all the nonsegmented pieces that make up the style and some variants based on the corresponding styles. The style number is derived from the SMR object and should be consistent with that in the intelligent analysis of computer-aided garment design CAD. In the nonsegmented piece object characteristic table, the key parameters for the characteristic assessment of the nonsegmented pieces and the variants of the corresponding nonsegmented pieces are included. The nonsegmented piece number is derived from the PTMR object and should be consistent with that in the computeraided garment design CAD intelligent analysis. The feature table of the prototypes includes the prototypes with various specifications from the key parameters of the prototype. The ID number of the prototype object characteristic table should be consistent with that of the PMR object in the computer-aided garment design CAD intelligent analysis. In this paper, the difference in specifications is extracted from the size specification table of the CAD intelligent analysis system via the interface. For the virtual reality model for surplus fabric removal and reuse without segmentation of cutting pieces of garment, the key parameters can also be extracted from the CAD intelligent analysis system through the interface.

5.6. System Integration Method. The computer-aided garment design CAD intelligent analysis and the integration method of CAD intelligent analysis system are generally divided into three stages from simple to complex. The packaging can be relatively simple, but it has great limitations. Integration is highly demanding for the skills of developers. At the same time, the cost of development and maintenance is also very high. The merging discussed in this paper is a method implemented through the interface. 
5.6.1. Interfaces for Registering Styles, Prototypes, and Nonsegmented Pieces in Garment CAD to the Intelligent Analysis of Computer-Aided Garment Design CAD. During the process of registering styles, prototypes, and nonsegmented pieces in the computer-aided garment design CAD intelligent analysis, the computer-aided garment design CAD intelligent analysis and two-way data exchange are required in the CAD intelligent analysis system on the one hand. On the other hand, it is necessary to extract the basic attribute information on the model, the nonsegmented table, and the prototype. For example, the materials, the author, and the subject are assigned to the master record of the model, as well as the corresponding attributes of the style master record (SMR), the parts master record (PTMR), and the prototype master record (PMR) objects. In addition, it is necessary to assign some attributes of the SMR objects, PTMR objects, and PMR objects to the corresponding attributes in the model, nonsegmented pieces, and prototypes, such as the pattern number, the nonsegmented piece number, and the prototype number. The location where the file is saved will be automatically adjusted accordingly.

\subsubsection{Creation of a Characteristic Table Interface of Objects.} In the creation of the object characteristic table in the virtual reality model for surplus fabric removal and reuse without segmentation of cutting pieces of garment, it is necessary to carry out data exchange with the intelligent analysis of computer-aided garment design CAD at the same time. In addition to the parameter information of the design, the style characteristic table of objects also contains the relationship between various cutting pieces that make up the model. In the creation of the style characteristic table of objects, the number of each nonsegmented piece needs to be extracted from the computer-aided garment design CAD intelligent analysis, and its consistency with the computer-aided garment design CAD intelligent analysis should be guaranteed.

\subsection{Support of System Integration for Garment Design Process.} System integration can provide excellent support for the garment development process of new products and the custom design process of the garment. The support for the new garment development process is mainly represented in the following aspects:

(1) Make it easy to acquire the development knowledge of a new garment

(2) Provide the function of searching in the existing style database, nonsegmented piece database, and prototype database

(3) Manage the development results of a new garment

In this way, designers can be truly freed from the arduous task of collecting the relevant knowledge, which is conducive to fully exerting the inspiration and creativity of designers. In addition, it can also consolidate the innovative knowledge of designers so as to enrich the virtual reality model for surplus fabric removal and reuse without segmentation of cutting pieces.
The primary support for the custom design process of the garment is described as follows.

(1) Save the result of custom design in the CAD intelligent analysis system from the nonsegmented pieces to the virtual reality model for surplus fabric removal and reuse through the interface

(2) Carry out the variant design of the prototype based on the data association in a simple way after the design is customized

(3) Make it convenient and efficient to search in the style database, the nonsegmented piece database for the management of the style database, the nonsegmented piece database, and the prototype database through the intelligent analysis of the computer-aided garment design $\mathrm{CAD}$

System integration can provide excellent support for design customization and variant design of the prototype.

\section{Application Examples}

The prototype variant design of a garment piece is used as an example to verify the principle described above. Based on system integration, a variant design of the prototype is carried out. The size of the specification table on the prototype size is extracted, and the characteristic object table from the nonsegmented pieces is introduced into the virtual reality model for surplus fabric removal and reuse for unified management based on the intelligent analysis of the computer-aided garment design CAD. In the field of garment design, a piece of garment can be broken down into several relatively independent pieces of the garment. For example, a coat is composed of a top, sleeves, stitching lines, pockets, zippers, and other parts. For each nonsegmented piece, the system determines the characteristic points that can represent its shape most properly first and then fit these characteristic points using several cubic B-splines to form the curve of the nonsegmented piece. Figure 12 is a schematic diagram of some nonsegmented pieces of the garment.

The application of nonsegmentation of cutting pieces in the virtual reality garment design for surplus fabric removal and reuse is also be reflected in the 3D display of the garment (Figure 13). The 3D nonsegmented pieces are transformed into a virtual reality model for surplus fabric removal and reuse, which allows the 3D representation of the fabric and implementation of $3 \mathrm{D}$ garment synthesis. The 3D garment can not only be viewed from any angle but also be modified with tools. For example, the pattern and color of the fabric can be modified and replaced at any time simply by using the material database in the system when trying on the garment. In this way, the design work can be carried out in a more intuitive, faster, and more convenient fashion. In addition, the hardness of the fabric can also be set to achieve stiffness or elegance in the garment style. Hence, through the virtual reality mode for surplus fabric removal and reuse based on the transformation of nonsegmented pieces, the garment can appear directly in front of people without being processed. 


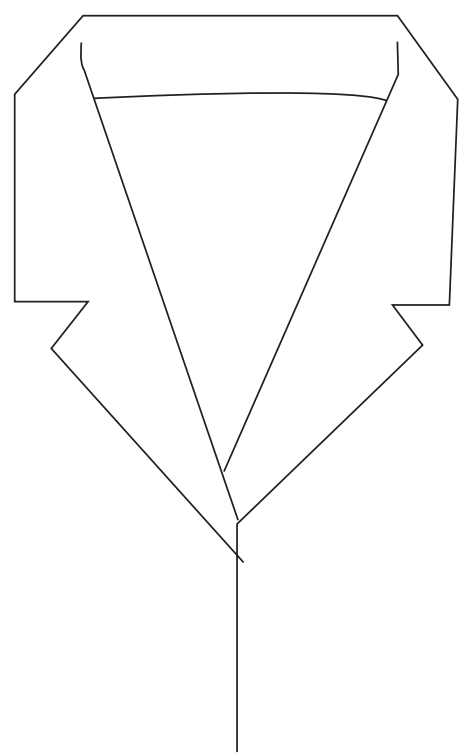

(a)

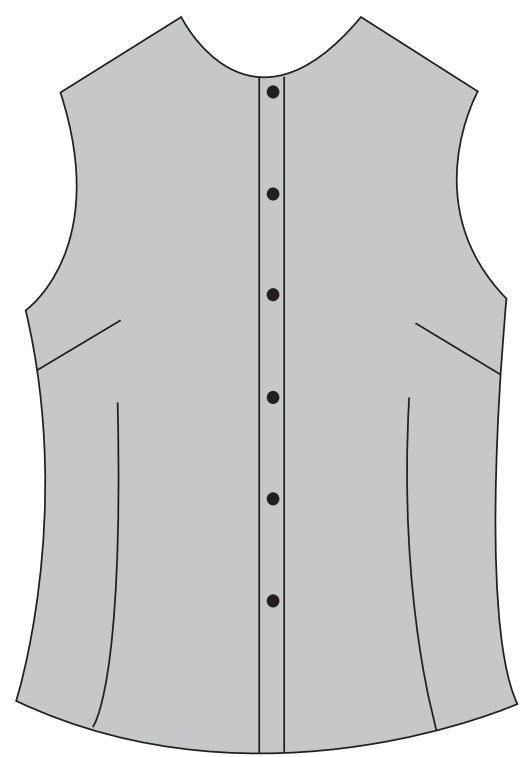

(b)

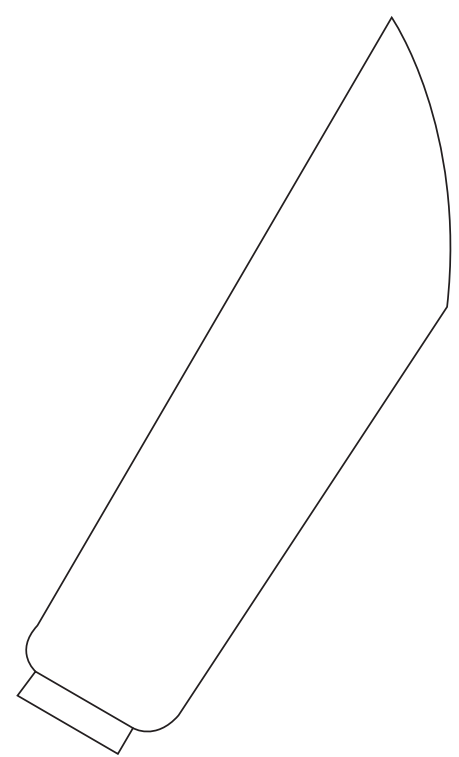

(c)

Figure 12: Garment without segmentation of cutting pieces. (a) Garment. (b) Cutting pieces of garment. (c) Sleeves.

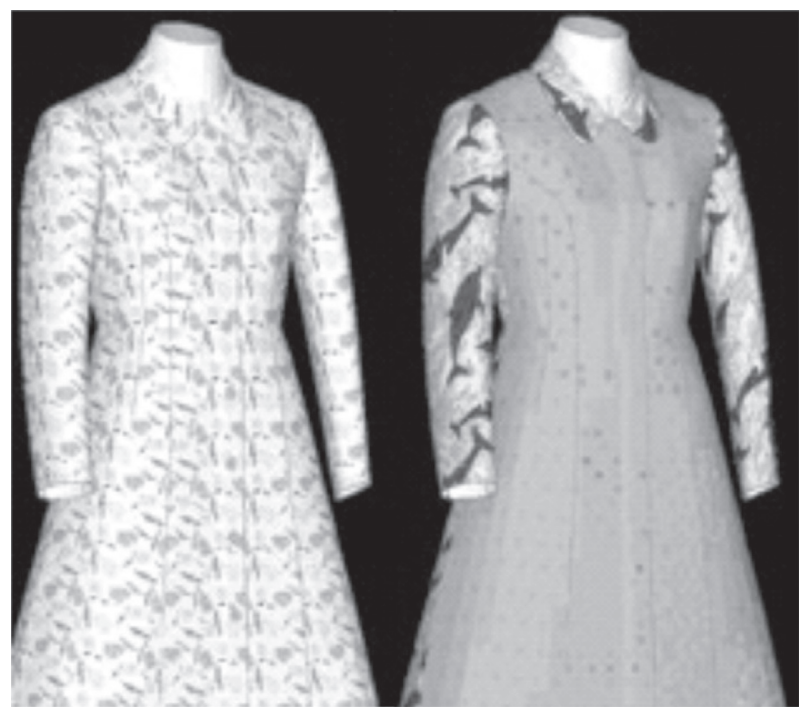

FIGURE 13: Virtual reality model for surplus fabric removal and reuse based on the transformation of nonsegmented pieces.

\section{Conclusions}

In order to reduce the time limit for personalized demand of customers, it is necessary to consider the combination of the features in the garment industry based on the CAD technology to improve the personalized design for customers. CAD technology is applied in the garment industry to manage the whole process of garment design and the design data information. At the same time, multiple aspects such as the garment styles, nonsegmented pieces, and even prototypes are managed accordingly. The virtual reality model for surplus fabric removal and reuse without segmentation of cutting pieces of the garment can implement the technology that allows the effective recycling of resources in the enterprises. In other words, the virtual reality model for surplus fabric removal and reuse without segmentation of cutting pieces has played a crucial role in the design of the modern garment industry and the intelligent application of CAD. With the rapid development of science and technology, the virtual reality model for surplus fabric removal and reuse without segmentation of cutting pieces has guided the garment industry into a new era.

\section{Data Availability}

The labeled dataset used to support the findings of this study is available from the corresponding author upon request.

\section{Conflicts of Interest}

The author declares no conflicts of interest.

\section{Acknowledgments}

This study was sponsored by Zhengzhou Technology and Business University.

\section{References}

[1] P. Mandal, "The role of ict in garment design \& textile education sector," Colourage, vol. 65, no. 8, pp. 29-30, 2018.

[2] H. T. Roman, "Garment design challenge," Technology and Engineering Teacher, vol. 79, no. 4, pp. 164-168, 2019.

[3] L. Valentine, "Garment design: facilitating generational change," Current Trends in Fashion Technology \& Textile Engineering, vol. 15, no. 2, pp. 392-405, 2018.

[4] A. Chen and S. Y. Ha, "Recycle garment design development using nature image," Journal of the Korea Fashion \& Costume Design Association, vol. 52, no. 7, pp. 120-128, 2018.

[5] Kim and Y. Sun, "Features of on aura tout vu's garment design-focusing on collections from 2002 f/w to 2015 s/s -,", 
Journal of the Korean Society Design Culture, vol. 26, no. 1, pp. 1-12, 2017.

[6] R. Liu and S. O. Fashion, "Research of futuristic style in garment design," Shandong Textile Economy, vol. 57, no. 2, pp. 345-354, 2017.

[7] S. Hashemi, H. Tann, and S. Reda, "Approximate logic synthesis using boolean matrix factorization: methodologies and cad," Approximate Circuits, vol. 15, no. 5, pp. 573-576, 2019.

[8] Matthias and Bohm, "Cad-design: die natur nachahmen," SMM Schweizer Maschinenmarkt, vol. 574, no. 6, pp. 914-923, 2018.

[9] S. W. Kielarova and P. Pradujphongphet, "Collaborative product design for product customization," An Industrial Case of Fashion Product, vol. 101, no. 3, pp. 620-627, 2020.

[10] M. C. Honigberg, B. S. Lander, V. Baliyan et al., "Preventive management of nonobstructive cad after coronary ct angiography in the emergency department," Journal of the American College of Cardiology: Cardiovascular Imaging, vol. 13, no. 2, pp. 437-448, 2020.

[11] X. Chen, Y. Chen, B. You, J. Xie, and H. Najjaran, "Detecting $6 \mathrm{~d}$ poses of target objects from cluttered scenes by learning to align the point cloud patches with the cad models," IEEE Access, vol. 68, no. 5, pp. 4157-4169, 2020.

[12] A. Scerrati, F. Trovalusci, A. Albanese et al., "A workflow to generate physical $3 \mathrm{~d}$ models of cerebral aneurysms applying open source freeware for cad modeling and 3d printing," Interdisciplinary Neurosurgery, vol. 17, pp. 1-6, 2019.

[13] Y. H. Kim and J. H. Lee, "A study on the related curriculum for preparing technical designers-focusing on garment and fashion related courses and graduate school curriculum in domestic university," Journal of the Korean Society Design Culture, vol. 6, no. 4, pp. 24694-24705, 2017.

[14] Fatma and Baytar, "Garment cad patternmaking with 3D simulations: impact of recurrent use of virtual prototypes on student's skill development," International Journal of Garment design, Technology and Education, vol. 64, no. 6, pp. 35-48, 2018.

[15] B. DeléPine, "Computer-aided design (cad) tools for bioproduction and biosensing pathway engineering," vol. 23, no. 10, pp. 110-115, 2017. 